Of those that had therapeutic levels of 6-TGN, 30/42 $(71 \%)$ were in clinical remission. $7 / 30(23 \%)$ were in total remission (clinical, biochemical [normal CRP/WCC], endoscopic and histological). 16/30 (53\%) were on Azathiopurine (AZA), 14/30 (47\%) were on mercaptapurine (6MP). 11/30 (37\%) were on combined therapy with biologics.

Of those that had non-therapeutic levels of 6-TGN, 19/32 $(59 \%)$ were in clinical remission. 5/19 (26\%) were in total remission. 12/19 (63\%) AZA. 7/19 (37\%) 6MP. 7/19 (37\%) were on combined therapy.

Of those with therapeutic levels of 6-TGN, 12/42 (29\%) were not in clinical remission.

6/12 (50\%) AZA, 6/12 (50\%) 6MP. 8/12 (75\%) were on combined therapy with biologics.

Of the cohort that had non-therapeutic levels of 6-TGN, $13 / 32(41 \%)$ were not in clinical remission. 6/13 (46\%) AZA, $7 / 13$ (44\%) $6 \mathrm{MP}$. 11/13 (85\%) were on combined therapy

Conclusion Our study so far suggests that, clinical remission rates were similar $(71 \%$ vs. $59 \%)$ for those who had therapeutic 6-TGN levels and for those who had not. It also shows that even without achieving therapeutic levels of 6TGN, 59\% of patients were still in clinical remission. Our study interestingly highlights that even with therapeutic levels of 6-TGN and with three quarters of patients on combined biologics $-29 \%$ were still not in remission. This preliminary study suggests interesting trends, that will be assessed further across the entire population $(n=426)$ and compared with European data.

\section{PWE-070 POST BIOLOGICAL INFUSION MONITORING; IS IT REALLY NECESSARY?}

Lisa Younge*, Ana Ibarra, Claire Healy, Marline Magsino, Klaartje Kok. Barts Health, London, UK

\subsection{6/gutjnl-2018-BSGAbstracts.202}

Introduction Biologic infusion services are seeing ever increasing activity. Facilitating this in a timely and safe manner with limited capacity is increasingly difficult.

Patients are monitored for infusion reactions following infliximab (IFX) and vedolizumab (VDZ) infusions, based on recommendations from the product manufacturers. Patients receiving IFX infusions are monitored in the unit for 2 hours following the infusion first four infusions, 1 hour following infusions 5-9 and $30 \mathrm{~min}$ for each infusion thereafter. For VDZ, a 2 hour observation period following the first 2 infusions is recommended.

The introduction of ustekinumab, licensed without a defined post infusion observation period, led us to consider the need for post infusion observation periods in other patient groups.

Methods We reviewed patient records of all patients receiving IFX and VDZ infusions in our clinic between September 2016 and September 2017 to identify infusion reactions and document when these occurred.

Results 1152 infusions of IFX and 330 infusions of VDZ were administered over the 12 month period. The total postinfusion observation time for these patients was 953 hours.

10 infusion reactions occurred $(0.9 \%)$, all in patients receiving IFX. 6/10 (60\%) occurred within 10 mins of starting IFX infusion (immediate), $2 / 10$ (20\%) later during the infusion (acute), and 1/10 (10\%) occurred 2 weeks after receiving the infusion (delayed). No infusion reactions occurred during the post-infusion observation period.

No infusion reactions occurred in patients receiving VDZ. Conclusions We analysed 953 post-infusion observation hours after 1152 IFX and VDZ infusions in our unit over a oneyear period. The total infusion reaction rate was $0.9 \%(10 /$ 1152). Of note, none of these occurred during the post-infusion period. The results from this large single centre retrospective study demonstrate that the risk of onset of adverse reactions to either IFX or VDZ during the post infusion observation period is very low. Patients who have not had a reaction during the infusion do not routinely need to stay in the unit for post infusion observation. We hope that by formally adapting this strategy we will better manage the increasing demands on our capacity, whilst still providing safe and appropriate care to our patients.

\section{PWE-071 ETROLIZUMAB INDUCTION IN MODERATE/SEVERE ANTI-TNF INTOLERANT/REFRACTORY (IR) UC: THE HICKORY OPEN-LABEL INDUCTION (OLI) TRIAL}

${ }^{1}$ Bu'hussain Hayee*, ${ }^{2}$ David Rubin, ${ }^{3}$ Brian Feagan, ${ }^{4}$ Young S Oh, ${ }^{4}$ Udayasankar Arulmani, ${ }^{5}$ Helen Tyrrell, ${ }^{4}$ Romeo Maciuca, ${ }^{5}$ Sarah Williams, ${ }^{4}$ Swati Tole, ${ }^{4}$ James Thommes, ${ }^{6}$ Lauren Peyrin-Biroulet. 'King's College Hospital, London, UK; ${ }^{2}$ University of Chicago Medicine, Chicago, USA; ${ }^{3}$ University of Western Ontario, London, Canada; ${ }^{4}$ Genentech, San Francisco, USA; ${ }^{5}$ Roche Products Ltd., Welwyn Garden City, UK; ${ }^{6}$ Université de Lorraine, Vandoeuvre-lès-Nancy, France

\subsection{6/gutjnl-2018-BSGAbstracts.203}

Introduction HICKORY OLI evaluated the safety and efficacy of etrolizumab (etro) via independent, centrally-read endoscopy, patient (pt)-reported outcomes, and inflammatory biomarkers in pts who are IR to aTNFs.

Methods Pts received etro $105 \mathrm{mg}$ injected SC every 4 weeks (14 week induction). Mayo clinical subscores (MCS) based on endoscopic score (ES), and pt-reported rectal bleeding (RB) and stool frequency (SF) were assessed at baseline (BL) and week 14. Clinical response: $\geq 3$ point and $30 \%$ reduction of MCS from BL and $\geq 1$ point decrease in $\mathrm{RB}$ or $\mathrm{RB} \leq 1$. Remission: MCS $\leq 2$ with individual subscores $\leq 1$ and $R B=0$. Endoscopic improvement: $\mathrm{ES} \leq 1$. RB remission: $\mathrm{RB}=0$; $\mathrm{SF}$ remission: $\mathrm{SF} \leq 1$ with $\geq 1$ point reduction from $\mathrm{BL}$. The $\%$ decline from $\mathrm{BL}$ in $\mathrm{RB}$ and $\mathrm{SF}$ at week 14 was also calculated.

Results HICKORY OLI enrolled 130 UC pts; 45\% had previously failed $>1$ TNF antagonist. BL disease activity included MCS score, 9.4; median C-reactive protein (CRP), 6.6 (95\% CI: $2.9,14.5) \mathrm{g} / \mathrm{dL}$; and median faecal calprotectin (FC), 1778 (95\% CI: 898, 3452) mg/kg.

At week 14, etro treatment was associated with clinical response in $50.8 \%$ of pts; remission in $12.3 \%$; ES $\leq 1$ in $23.9 \%$; $\mathrm{RB}$ remission in $52.3 \%$; and $\mathrm{SF}$ remission in $35.4 \%$. $43.9 \%$ of pts had $\geq 1$ point improvement from $\mathrm{BL}$ in the $\mathrm{ES}$ score, and improved ES scores were associated with increased rates of $\mathrm{RB}$ and $\mathrm{SF}$ remission. Among pts with $\mathrm{ES}=0,100 \%$ reported $\mathrm{RB} \leq 1$, and $90 \%$ reported $\mathrm{SF} \leq 1$ (table 1). Pts who achieved either $\mathrm{SF}$ or $\mathrm{RB}$ remission or ES $\leq 1$ also demonstrated $>50 \%$ geometric mean reduction in CRP $(\mathrm{BL} \geq 2.87 \mathrm{mg} / \mathrm{L})$ and $>70 \%$ geometric mean reduction in $\mathrm{FC}$. Conclusions TNF antagonist-experienced pts with moderatesevere UC and high disease burden treated with open label etro for 14 weeks achieved clinically meaningful clinical response and remission and endoscopic improvement. Pts who had a decline in $E S \geq 1$ achieved higher rates of $\mathrm{RB}$ and $\mathrm{SF}$ 